\title{
Cardioversion of atrial fibrillation of recent onset with
} flecainide

\author{
B. Carr, K. Hawley and K.S. Channer \\ Department of Cardiology, Royal Hallamshire Hospital, Glossop Road, Sheffield S10 2JF, UK
}

\begin{abstract}
Summary: Twenty-eight patients with atrial fibrillation of less than 1 month's duration were given a single intravenous bolus of flecainide $(2 \mathrm{mg} / \mathrm{kg})$. Nineteen patients $(68 \%)$ were successfully converted to sinus rhythm. Of the 19 patients with atrial fibrillation of less than 72 hours duration, $18(95 \%)$ were successfully converted. Failure to cardiovert was associated with cardiomegaly on chest X-ray and larger left atrial diameter measured echocardiographically. Of the 9 patients who failed to convert, 2 had biochemical evidence of thyrotoxicosis and chronic obstructive pulmonary disease. Of the 19 patients successfully cardioverted, the atrial fibrillation complicated acute myocardial infarction in 3 cases and a pulmonary embolism in one. Six cases occurred post-operatively and in 3 out of 6 the patient was ventilated. No side effects were observed.

The use of intravenous flecainide should avoid the need for electrical DC conversion even in sick patients. Failure of conversion is associated with covert underlying predisposing factors and duration of atrial fibrillation of more than 72 hours.
\end{abstract}

\section{Introduction}

Atrial fibrillation is common and its incidence increases with age. ${ }^{1}$ It is an inefficient rhythm because its onset is associated with the loss of synchronous atrio-ventricular contraction; cardiac output may be reduced by up to $50 \%$ in some subjects. ${ }^{2}$ It is associated with a significant increase in systemic embolism. ${ }^{3}$ Thus, cardioversion of all patients with atrial fibrillation should be considered. Where there is a persisting underlying cause (for example mitral valve disease) cardioversion is unlikely to be successful in the long term and management in these cases is aimed at control of the ventricular rate.

The most effective method of cardioversion is electrical DC counter shock. However, this technique requires a general anaesthetic and therefore carries a measurable morbidity. It requires the skills of an anaesthetist, which may be a limited resource. Pharmacological cardioversion of atrial fibrillation has been disappointing. Digoxin given intravenously and oral quinidine, for example, were successful in less than $50 \%$ of cases, ${ }^{4-6}$ whereas verapamil was successful in less than $10 \% .^{7}$ More recently, flecainide, a class 1c antiarrhythmic drug, has been shown to be much more effective in converting supraventricular arrhyth-

Correspondence: K.S. Channer, M.D., M.R.C.P. Accepted: 5 February 1991 mias including atrial fibrillation. ${ }^{7,8}$ Previous studies have suggested that long standing atrial fibrillation is less successfully cardioverted. We report our experience with intravenous flecainide in patients with atrial fibrillation of recent onset.

\section{Methods}

Twenty-eight consecutive patients with atrial fibrillation of less than one month's duration took part in the study. All were in-patients at the time and had been selected for attempted electrical cardioversion. None had clinical evidence of heart failure nor any clinically apparent untreated underlying conditions (for example thyrotoxicosis; mitral valve disease) likely to render cardioversion ineffective. All had a chest X-ray and a 12-lead electrocardiograph and over half (19) had echocardiography.

We did not routinely anticoagulate patients prior to cardioversion if the atrial fibrillation was of short duration (less than 72 hours). However, patients with atrial fibrillation for more then 72 hours had intravenous heparin, 30,000 units per 24 hours for 72 hours, prior to flecainide or DC cardioversion to minimize the risk of thromboembolism following a return to sinus rhythm.

An intravenous infusion of flecainide $(2 \mathrm{mg} / \mathrm{kg})$ was given over 10 to 15 minutes with continuous electrocardiographic monitoring. The time taken 
to cardioversion was noted. Patients who failed to convert with flecainide subsequently had elective DC cardioversion.

Appropriate statistical methods were used to assess differences between subgroups.

\section{Results}

Twenty of the 28 patients were male. The average age of the group was 61 years (range 20-79). All had atrial fibrillation and one also had episodes of atrial flutter. Flecainide was successful in 19 patients $(68 \%)$. Eighteen of these 19 had atrial fibrillation for less than 72 hours and only one patient with atrial fibrillation of less than 72 hours failed to cardiovert. The success rate in this early group was $95 \%$. In all cases the cardioversion occurred within 2 hours of the start of the infusion and mean time to cardioversion was 24 minutes. Only one of the 9 patients with atrial fibrillation of duration more than 72 hours and less than one month converted to sinus rhythm and this occurred at 24 hours, which may not have been a direct effect of the drug. The infusion was well tolerated by all patients and no side effects were reported. In one case a 2 second asystolic pause occurred during the infusion but this required no treatment. No embolic phenomena were seen in either group.

\section{Cause of atrial fibrillation}

The causes of the atrial fibrillation in the early and late groups are shown on Table I. Hypertension and ischaemic heart disease were commonly associated with atrial fibrillation in both groups. In the late group, 2 patients were found to have biochemical evidence of thyrotoxicosis despite being clinically euthyroid; both failed to cardiovert. In 3 patients chronic obstructive airways disease or emphysema were associated with the atrial fibrillation and flecainide was unsuccessful in all these cases also. In the early group, 6 post-operative patients developed atrial fibrillation, ( 1 as a consequence of acute pulmonary embolism). Three of these patients were very ill and ventilated at the time and two developed atrial fibrillation as part of a septicaemic episode. Four patients developed atrial fibrillation within 48 hours of an acute myocardial infarction and one patient developed atrial fibrillation during treadmill testing for angina. In 2 patients high alcohol intake was associated with the onset of atrial fibrillation. All patients in the early group, except one with atrial fibrillation following a sub-endocardial myocardial infarction, were successfully cardioverted with an intravenous bolus of flecainide. In all cases the cardioversion was associated with haemodynamic improvement even in those patients on ventilators.
Table I Cause of atrial fibrillation in patients with duration of less than 72 hours (early) and more than 72 hours, less than one month (late).

\begin{tabular}{lll}
\hline & $\begin{array}{l}\text { Early } \\
n=19\end{array}$ & $\begin{array}{l}\text { Late } \\
n=9\end{array}$ \\
\hline $\begin{array}{l}\text { Age median; range years } \\
\text { Hypertension }\end{array}$ & $59 ; 20-79$ & $69 ; 54-75$ \\
$\begin{array}{l}\text { Ischaemic heart disease } \\
\text { Chronic obstructive }\end{array}$ & 6 & 2 \\
pulmonary disease & 1 & 4 \\
$\begin{array}{l}\text { Thyrotoxicosis } \\
\text { Alcohol }\end{array}$ & 0 & 3 \\
$\begin{array}{l}\text { Post-operative } \\
\text { Acute pulmonary embolism }\end{array}$ & 2 & 2 \\
Acute myocardial infarction & 4 & \\
\hline
\end{tabular}

In no patient did flecainide cause haemodynamic deterioration.

Ten patients $(53 \%)$ in the early group in which cardioversion with flecainide was successful were taking predominantly cardioactive drugs including digoxin (3), calcium antagonists (6) and beta adrenoreceptor blockers (3), compared with 6 $(67 \%)$ patients in the late group (digoxin 4 ; beta adrenoreceptor blockers 1; amiodarone 1). Nine of $19(47 \%)$ of the early group and 3 of $9(33 \%)$ of the late group were not taking any form of concomitant anti-arrhythmic therapy $(P=$ not significant, exact test).

Details of the initial heart rate, chest X-ray and echocardiograms are shown in Table II. Significantly more patients in the early group had normal cardiac size on chest X-ray and smaller average left atrial diameter on echocardiography.

\section{Discussion}

In this study we have found that a single intravenous bolus of flecainide ( $2 \mathrm{mg} / \mathrm{kg}$ ) was successful in cardioverting $95 \%$ of patients presenting with atrial fibrillation within 3 days of its onset. It was successful even in very ill patients who were ventilated or who had developed atrial fibrillation as a complication of myocardial infarction or pulmonary embolism. It was well tolerated and caused no haemodynamic deterioration - rather cardioversion was associated with haemodynamic improvement, presumably related to the return of atrioventricular synchrony. ${ }^{2}$

Although longstanding atrial fibrillation is associated with thromboembolic phenomena we did not formally anticoagulate our patients if the arrhythmia was of short duration. We feel justified in this policy since no embolic phenomena were seen.

Previous studies have shown that the efficacy of 
Table II Details of initial heart rate, chest X-ray and echocardiography in patients with atrial fibrillation of less than 72 hours and more than 72 hours duration

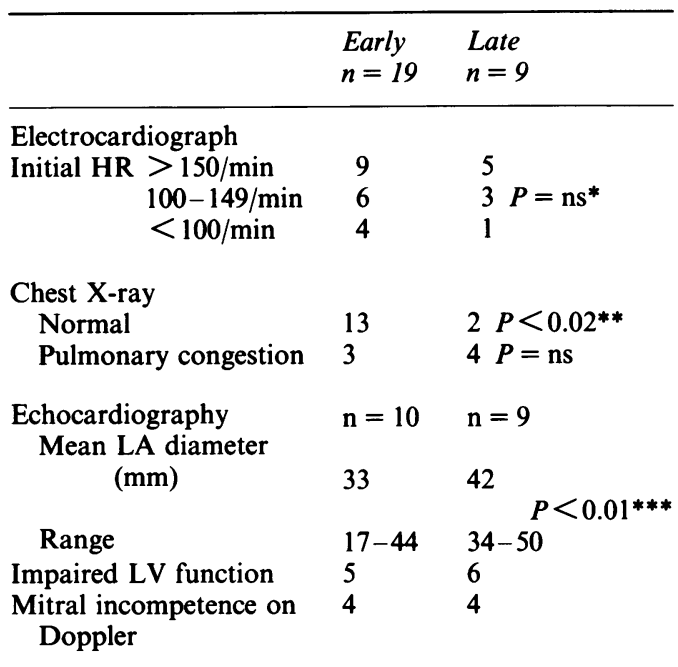

*Chi square test; ${ }^{* *}$ Exact test; ${ }^{* * * t}$ test; HR = heart rate; $\mathbf{L A}=$ left atrial; $\mathbf{L V}=$ left ventricular.

flecainide falls with duration of the arrhythmia. Goy et al. ${ }^{8}$ in a study involving 69 patients with atrial fibrillation, found a cardioversion rate of $79 \%$ in patients of less than 10 days duration compared with $38 \%$ in patients with atrial fibrillation of longer duration. Suttorp et al., ${ }^{7}$ in a study of 32 patients, found a conversion rate of $86 \%$ in patients with the arrhythmia of less than 24 hours duration, compared with $40 \%$ in patients with atrial fibrillation of more than 24 hours duration. Our data shows a cut off at 72 hours duration, with a very low conversion rate of only $14 \%$ in patients with atrial fibrillation of longer duration.

Other factors which have been associated with failure of cardioversion with flecainide include increased left atrial dimension. ${ }^{8}$ This was also shown in our study. However, we believe atrial fibrillation is associated with an increase in left atrial dimension as a consequence of the arrhythmia itself. Indirect evidence to support this is provided by our echo data which show that those patients with atrial fibrillation of less than 72 hours duration have smaller left atrial diameter than those with atrial fibrillation more than 72 hours duration. We suggest that the longer the duration of atrial fibrillation the larger the left atrial dimension and the less likely that successful cardioversion will be achieved. For this reason longstanding atrial fibrillation is rarely successfully cardioverted. Following cardioversion from atrial fibrillation atrial contraction gradually improves over one month as detected by Doppler measures of transmitral blood flow, ${ }^{9}$ suggesting that left atrial tone takes some time to return to normal. Because of the relationship between atrial fibrillation and atrial dilatation it is important to attempt cardioversion as soon as possible once the arrhythmia has developed. However, it is equally important to correct other underlying predisposing conditions. In the late group 2 patients had biochemical evidence of thyrotoxicosis (normal T4, T3 but suppressed high sensitivity TSH) and both have had radioiodine treatment, and 3 patients had longstanding chest disease. Under these circumstances cardioversion is less likely to be successful because its precipitating underlying cause is still present.

The use of flecainide for ventricular arrhythmias following acute myocardial infarction has recently been discouraged because of worries over its safety. These worries concern the known proarrhythmic effects of class 1 agents especially in the presence of impaired left ventricular function. In the CAST study $^{10}$ the drug was given chronically in its oral form. An intravenous bolus of the drug is less likely to be associated with morbidity and has been used extensively and been found to be well tolerated and safe, as in our study.

In conclusion we have found intravenous flecainide $(2 \mathrm{mg} / \mathrm{kg})$ to be a safe and effective treatment for cardioversion of atrial fibrillation of less than 72 hours duration. Its use should avoid the need for DC cardioversion in these cases. Failure of cardioversion is associated with covert underlying predisposing factors and duration of atrial fibrillation of more than 72 hours.

\section{References}

1. Kannel, W.B., Abbott, R.D., Savage, D.D. \& McNamara, P.M. Epidemiologic features of chronic atrial fibrillation. The Framingham Study. $N$ Engl $J$ Med 1982, 306: $1018-1022$.

2. Channer, K.S. \& Jones, J.V. Atrial systole; its role in normal and diseased hearts. Clin Sci 1988, 75: 1-4.

3. Wolf, P.A., Kanner, W.B., McGee, D.L., Meek, S.L., Bharacha, N.E. \& McNamara, P.M. Duration of atrial fibrillation and imminence of stroke. The Framingham Study. Stroke 1983, 14: 664-667.

4. Weiner, R., Bassan, M.M., Jarchovsky, J., Iusim, S. \& Plavnick, L. Clinical course of acute atrial fibrillation treated with rapid digitalisation. Am Heart J 1983, 105: 233-237.

5. Kory, R.C. \& Meneely, G.R. Cardiac output in auricular fibrillation with observations on the effects of conversion to sinus rhythm. J Clin Invest 1951, 30: 653.

6. Hecht, H.H., Osher, W.J. \& Samuels, A.J. Cardiovascular adjustments in subjects with organic heart disease before and after conversion of atrial fibrillation to normal sinus rhythm. J Clin Invest 1951, 30: 647. 
7. Suttorp, M.J., Kingma, J.H., Lie-A-Huen, L. \& Mast, E.G Intravenous flecainide versus verapamil for acute conversion of paroxysmal atrial fibrillation or flutter to sinus rhythm. Am J Cardiol 1989, 63: 693-696.

8. Goy, J.J., Kaufman, U., Kappenberger, L. \& Sigwart, U. Restoration of sinus rhythm with flecainide in patients with atrial fibrillation. Am J Cardiol 1988; 62: 38D-40D.

9. Lipkin, D.P., Frenneaux, M., Stewart, R., Joshi, J., Lowe, T. \& McKenna, W.J. Delayed improvement in exercise capacity after cardioversion of atrial fibrillation to sinus rhythm. $\mathrm{Br}$ Heart J 1988, 59: 572-577.
10. CAST investigators. Preliminary report: effect of encainide and flecainide on mortality in a randomised trial of arrhythmia suppression after myocardial infarction. $N$ Engl $J$ Med 1989, 321: 406-412. 